\title{
An Attempt at Large Eddy Simulation for Combustor Modeling
}

\author{
Leiyong Jiang ${ }^{*}$ and Ian Campbell
}

Gas Turbine Laboratory, Institute for Aerospace Research, the National Research Council of Canada, 1200 Montreal
Road, M-10, Ottawa, Ontario, Canada, KlA OR6

\begin{abstract}
Large eddy simulation (LES) is a promising method for numerical simulation in combustion systems. A LES attempt in a model combustor has been made, and a few important issues related to grid size, inflow condition, wall boundary conditions, physical sub-models and data sampling are discussed. Some of the numerical results are presented and compared with a comprehensive experimental database, which indicates that LES can provide reasonable predictions for the mean axial velocity and temperature distributions inside the combustion chamber. However, in order to make LES a valuable and cost-effective tool in the development of advanced combustion systems, some fundamental questions remain to be addressed and more validation efforts are required. Moreover significant computing power is required for LES to capture both the high and low frequencies of interest in the present turbulent reacting flow.
\end{abstract}

Keywords: combustion, combustor modeling, gas turbine, large eddy simulation, thermal boundary, turbulence modeling.

\section{INTRODUCTION}

Large eddy simulation (LES) is considered as a promising method for simulating turbulent reacting flows, such as gas turbine combustors where large vortices dominate the flow field [1-2]. With this approach, the large, energy-carrying eddies are directly resolved (without the need of physical models), whereas the small, sub-grid-scale (SGS) eddies are modeled. In principle, LES is more universal than the Reynolds-averaged Navier-Stokes (RANS) method; because the small-scale eddies tend to be more isotropic and homogeneous than the large ones and thus more amenable to universal modeling. Furthermore, the modeled SGS stresses only contribute a small fraction of the total turbulent stresses in general.

Combustion LES appeared in the literature a little more than a decade ago and the recent progress has been reviewed by Pitsch [1]. It was concluded that the full predictive potential has not been realised, and many fundamental questions of combustion LES have to be addressed. James and his co-workers [2] presented an overview of their recent achievements in LES as a design tool for gas turbine combustion systems. A number of SGS and LES combustion models were successfully implemented into their in-house capabilities. Validation cases from a simple lid-driven cavity flow to a complex gas turbine combustor were performed, which highlighted the predictive capabilities of LES. They pointed out that more validation and verification efforts are required to assess the limitations and accuracy of physical sub-models, and the turnaround time is a key issue for LES to be accepted as a practical design tool in industry.

*Address correspondence to this author at the Gas Turbine Laboratory, Institute for Aerospace Research, the National Research Council of Canada, 1200 Montreal Road, M-10, Ottawa, Ontario, K1A 0R6, Canada; Tel: 613993-9235; Fax: 613-952-7677; E-mail: leiyong.jiang@nrc-cnrc.gc.ca
There are a fairly large number of publications in LES related to gas turbine combustion such as [2-9]. However, simulations with detailed quantitative validation against well-defined experimental results are rare, with few exceptions.

LES studies in a swirl-stabilized (methane-air diffusion flame) model combustor were carried out by James et al., [2], where 1.1 million grid points were used. Reasonable agreement between the numerical and experimental results was illustrated for mean axial velocity profiles at three downstream cross-sections. The filtered density function (FDF) combustion model showed better performance than the probability density function (PDF) model. For the temperature predictions at these three sections, the results from five model options were compared with the experimental data. Unfortunately, none of them could provide adequate prediction for all three sections.

Large eddy simulation of a Rolls-Royce production gas turbine combustor with liquid jet fuel was also investigated by James et al., [2]. The computational domain, including a swirler, a fuel nozzle, primary and dilution holes, cooling elements, an annuli and the combustor liner, consisted of $\sim 1.0$ million cells. The localized dynamic SGS model and eddy breakup combustion model were employed to calculate SGS turbulent transfer and chemical reactions respectively. In comparison with the measured mean temperature profile at the combustor exit, it was found that the predicted temperature profiles from the LES of the liner plus annuli case and the RANS of the liner-only case were similar and close to the experimental data, slightly superior over the LES of liner only.

Large eddy simulations with 1.3 million cells in a coaxial model combustor fuelled with methane (diffusion flame) were carried out by Mahesh et al., [3], where a mixturefraction/progress-variable approach was employed to model chemical reactions. An acceptable agreement between the 
predicted results and experimental data was observed at three downstream sections for mean and rms of axial velocity, mean temperature, mixture fraction, progress variable (sum of mass fractions of $\mathrm{CO}_{2}$ and $\mathrm{H}_{2} \mathrm{O}$ ) and mass fraction of $\mathrm{CO}$.

To provide a benchmark database for the evaluation and development of various physical models, a series of experiments were performed on a diffusion flame model combustor at the National Research Council of Canada. The comprehensive results include mean and fluctuating velocity components, mean temperature, wall temperature, radiation heat flux, as well as species concentrations [10].

The objective of the present work is to assess the performance of LES, as an engineering tool, in the model combustor. A few important factors affecting LES results are discussed, including grid size determination, inflow perturbation, wall boundary conditions, physical sub-models, and data sampling. Since the combustor geometry is simple and a comprehensive database is available, the assessment is relevant. Moreover, the combustion process in diffusion flames is mainly controlled by turbulent mixing [11, 12]; therefore the uncertainty or errors of LES related to combustion modeling is minimized.

\section{EXPERIMENTAL MEASUREMENTS}

A schematic diagram of the model combustor is shown in Fig. (1), including the fuel and air inlet section, combustion chamber, and contracted exhaust section (all dimensions are in $\mathrm{mm}$ ). Air entered the combustion chamber around a disc flame-holder, while fuel was fed through the center of the bluff body. The diameter of the fuel inlet tube was $8.45 \mathrm{~mm}$. To have a flat-top velocity profile at the air inlet, a large converging plenum was installed upstream of the fuel/air inlet section. This plenum had a honeycomb section, and accommodated air, fuel and LDA (laser Doppler anemometer) seeding pipe lines. These portions of the experimental rig are not shown in Fig. (1), nor included in the computational domain for the present study.

The test rig was mounted on a three-axis traversing unit with an accuracy of $\pm 100 \mu \mathrm{m}$. The Reynolds number was $1.9 \times 10^{5}$ for the airflow based on the entry velocity and flame-holder diameter, and for the fuel flow it was $3.0 \times 10^{4}$. Fuel used in the experiments was commercial grade propane, and dry air was delivered from a shop air supply. Both air and fuel flows were controlled by Sierra Side-Trak massflow controllers with $2 \%$ accuracy of full scale (fuel 100 $1 / \mathrm{min}$ and air $2550 \mathrm{l} / \mathrm{min}$ ).

To reduce the heat losses through walls, a 25.4-mm thick fibre blanket of $\mathrm{Al}_{2} \mathrm{O}_{3}$ was wrapped around the combustion chamber. Four narrow slots were cut into the blanket to allow physical and optical access to the chamber interior. Interchangeable sets of stainless steel and fused silica windows were used for physical probing using gas sampling probes and thermocouples, as well as for optical probing using the LDA. The viewing area of the windows measured $17 \mathrm{~mm}$ in width and $344 \mathrm{~mm}$ in length. The combustor geometry was relatively simple compared with practical combustion systems, but fundamentally similar and pertinent to the modeling of complex combustion systems.

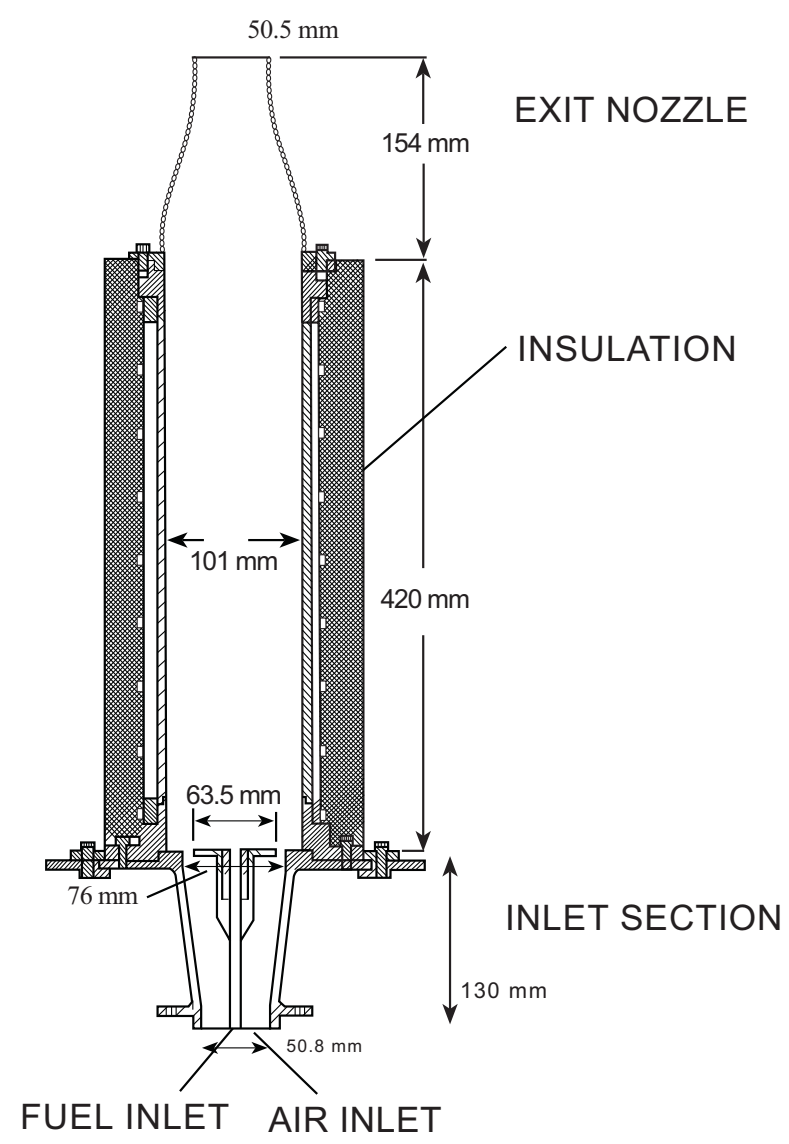

Fig. (1). The model combustor.

Measurements of velocity were made using both a 2- and 3 -component LDA system operating in a 30-degree-offset back scattering mode. In the lower section of the combustion chamber, limited optical access forced the use of a single fibre optic head to measure axial and tangential velocities. In the upper section of the chamber, the 3-component LDA system was used to measure all three velocity components. To ensure the statistics accuracy of velocity measurements, a total of 20,000 samples were collected and processed at each measurement location in general. The probe volume size of the LDA systems was $0.12 \mathrm{~mm}$ in diameter and $0.6 \mathrm{~mm}$ in length.

Gas temperatures were acquired using an uncoated 250$\mu \mathrm{m}$ diameter, type " $\mathrm{S}$ " thermocouple supported by a twinbore ceramic tube. Thermocouples embedded in and flush with the combustor wall were used to measure the wall temperature. Gas species measurements were made with a sampling probe connected to a Varian Model 3400 Gas Chromatograph. The major species measured included $\mathrm{CO}$, $\mathrm{CO}_{2}, \mathrm{C}_{3} \mathrm{H}_{8}$ and $\mathrm{O}_{2}$. NOx and $\mathrm{NO}$ were collected through the same probe but analysed using a Scintrix NOx analyser.

\section{NUMERICAL SIMULATIONS}

Axi-symmetric, turbulent, reacting flows were considered in the present study, and a commercial software package, Fluent, was used as a platform for all numerical studies. The computational domain, governing equations, selected physical sub-models, boundary conditions, and solution methods are described in the following sub-sections. 


\subsection{Computational Domain and Mesh}

The computational domain covered the whole combustor flow field from the fuel/air inlets to the exhaust exit (see Fig. 1). In a previous 2-D RANS study on this combustor [12], the Reynolds stress turbulence model (RSM), eddydissipation combustion model, and optimized turbulent Prandtl and Schmidt number were employed, and the turbulence kinetic energy and shear stress were fairly well predicted in comparison with the experimental database. Based on these results, the local energetic turbulence length scales [13] in the combustor were estimated and used to generate the mesh for the present LES. Fine grids were laid in the shear layers between the recirculation region and fuel/air jets, and in the combustion chamber. The mesh size in the radial direction is $0.4 \mathrm{~mm}$ in the shear layer and 0.6 $\mathrm{mm}$ in other regions of the combustion chamber, less or equal to the length of the LDA probe volume. Along the circumferential direction, a total of 60 planes were generated. The $\mathrm{y}^{+}$values at all walls of the computational domain varied in the range of $1-30$. A total of 1.93 million hexahedral cells were used for all simulations.

Unlike RANS simulations, which will generally produce mesh-independent results as soon as the mesh is sufficiently refined, the model itself in LES is a function of the grid cell size and the discretization and model errors can interact dynamically. This issue becomes even more challenging due to the significant computing time required to undertake systematic mesh-dependence studies.

Recently, Gant [14] has surveyed a number of practical methods for mesh-independence assessment in LES. One among them is also proposed by the authors, and used in the present study. That is, the mesh-independence is assessed by a grid factor $I, R_{1}$, defined as the ratio of the local grid size to the local energetic turbulence length scale:

$$
R_{1}=\Delta / l=V^{1 / 3} /\left(k^{3 / 2} / \varepsilon\right)
$$

where $\Delta$ is the grid size, $l$ is the energetic turbulence length scale, and $V$ stands for the cell volume.

This method has been applied to a tunnel fire and a buoyancy-opposed wall-jet, and it is found that the ratio needs to be less than $1 / 12(0.083)$ for the LES to be well resolved [14]. Note that this criterion does not apply in near wall viscous sub-layer regions.

Fig. (2) shows the contours of the grid factor $I$ at a longitudinal plane of the combustor, obtained from a 3-D RANS simulation with the mesh created for LES and the Reynolds stress turbulence model. In Fig. (2), the axis of X is along the combustor centerline with its origin at the backward surface of the bluff-body flame-holder, while the axis of $\mathrm{R}$ is in the combustor radial direction. Fig. (2) has shown that the grid factor I is less than 0.08 in most regions of the combustion chamber.

The effect of grid resolution on LES in a helicopter combustor was also investigated by Boudier et al., [15], where the Smagorinsky SGS turbulent viscosity model and one-step chemistry were employed. It was found that the mean flow parameters (velocity, temperature and reaction rate) were almost insensitive to mesh sizes in a range of 1.2 to 44 million cells. The mesh size used in the present study is larger than those used in [2-3] for diffusion flame model combustors. In short, in terms of current understanding of LES in engineering applications, it is considered that the mesh size is reasonable for this engineering exercise.

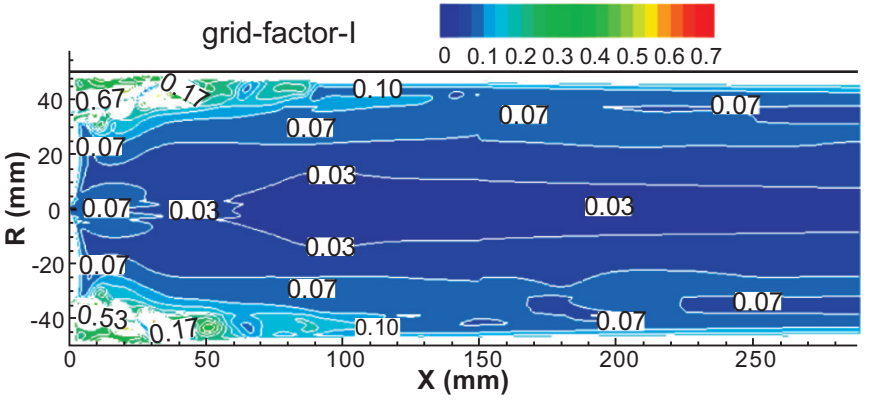

Fig. (2). Grid factor $I, R_{1}$, estimated from RANS simulation.

\subsection{Filtered Governing Equations}

The incompressible conservation equations of mass, momentum, species and total enthalpy filtered by the finitevolume discretization may be expressed as [16]:

$$
\begin{aligned}
& \frac{\partial \rho}{\partial t}+\frac{\partial}{\partial x_{i}}\left(\rho \bar{u}_{i}\right)=0 \\
& \frac{\partial}{\partial t}\left(\rho \bar{u}_{i}\right)+\frac{\partial}{\partial x_{j}}\left(\rho \bar{u}_{i} \bar{u}_{j}\right)=\frac{\partial}{\partial x_{j}}\left(\frac{\partial \bar{\sigma}_{i j}}{\partial x_{j}}\right)-\frac{\partial \bar{p}}{\partial x_{i}}-\frac{\partial \tau_{i j}}{\partial x_{j}} \\
& \frac{\partial}{\partial t}(\rho \bar{\varphi})+\frac{\partial}{\partial x_{i}}\left(\rho \bar{u}_{i} \bar{\varphi}\right)=\frac{\partial}{\partial x_{i}}\left(\rho D \frac{\partial \bar{\varphi}}{\partial x_{i}}\right)-\frac{\partial M_{i}}{\partial x_{i}}+\bar{S}
\end{aligned}
$$

In the above equations, filtered variables are denoted by an over-bar, $\bar{\sigma}_{i j}$ is the filtered stress tensor due to molecular viscosity, $\bar{\varphi}$ stands for species or total enthalpy, $\bar{S}$ is the source term, $\mu$ and $\mathrm{D}$ are the molecular viscosity and diffusivity, and $\tau_{\mathrm{ij}}$ and $\mathrm{M}_{\mathrm{i}}$ are the SGS stress and scalar flux of species or total enthalpy, respectively.

\subsection{Physical Sub-Models}

For closure of the above governing equations, the SGS stress and scalar flux have to be calculated by a selected SGS model. The source term in Eq. (4) can be obtained from a selected combustion model for species, and it may include viscous heating and radiation heat transfer for total enthalpy.

The dynamic SGS kinetic energy model proposed by Kim and Menon [17] was chosen to model SGS turbulence transfer in the combustor. Unlike the essentially algebraic Smagorinsky-Lilly models, the transport equation of the SGS turbulence kinetic energy was resolved throughout the flow field. Therefore, the underlying local equilibrium assumption between the transferred energy through the grid-filter scale and the dissipation of kinetic energy at small sub-grid scales was removed.

For the SGS scalar flux, the Reynolds analogy similar to RANS simulation was applied. In RANS simulations, the turbulent Prandt//Schmidt number is commonly assumed to 
be close to 0.7 . However, in LES this value is different. Moin et al., [18] generalized a dynamic SGS model. The model was applied to a decaying isotropic turbulent flow, and the LES results were in excellent agreement with experimental data and direct numerical simulations. The expression of the SGS turbulent Prandtl number was evaluated by using direct numerical simulations (DNS). It was found that the SGS turbulent Prandtl number could be approximated by 0.6 for two compressible isotropic turbulent flows, 0.5 for a homogenous shear flow with scalar gradient in the normal direction and molecular Prandtl number of 0.7, and 0.5 in the central region of a channel flow.

For the LES of a turbulent piloted jet diffusion flame, Pitsch and Steiner [19] evaluated the SGS eddy viscosity and diffusivity by applying a dynamic SGS model. The resulting SGS Schmidt number profiles were obtained at six crosssections from 7.5 to 75 nozzle-diameters downstream. The data clearly indicated that a constant value of 0.4 is a good approximation throughout the whole domain.

Based on the above studies, as an approximation, a SGS Prandtl/Schmidt number of 0.45 was used in the present work.

For the combustion modeling in the combustor, the eddydissipation (EDS) model was employed since it has been widely accepted in diffusion flame modeling [2, 11, and 20]. With this model, a one-step global chemical reaction was assumed,

$\mathrm{C}_{3} \mathrm{H}_{8}+5\left(\mathrm{O}_{2}+3.76 \mathrm{~N}_{2}\right)$

$=3 \mathrm{CO}_{2}+4 \mathrm{H}_{2} \mathrm{O}+5 \times 3.76 \mathrm{~N}_{2}$

and the reaction rate was governed by turbulent mixing. For the application to LES, the turbulent mixing rate, $\varepsilon / \mathrm{k}$, in the original work of Magnussen and Hjertager [21] has been replaced by the SGS mixing rate $\tau_{s g s}=\sqrt{2 \bar{S}_{i j} \bar{S}_{i j}}$ with $\bar{S}_{i j}$ the resolved strain rate tensor [16].

Radiation between the combustor wall and gaseous mixture in the combustion chamber was neglected since NOx prediction was not considered in the simulation. The previous RANS benchmarking on radiation prediction [22] has indicated that the effect of radiation on the flow field is minor, particularly to the velocity field.

Polynomials were used to calculate the specific heat of species as a function of temperature. The polynomials determined from reference [23] were used, where the chemical dissociation was considered. For other thermal properties of the mixture such as molecular viscosity, thermal conductivity and diffusivity, the values of air at 900 $\mathrm{K}$ were used.

\subsection{Boundary Conditions}

The fuel (propane) mass flow rate was $16.2 \mathrm{~g} / \mathrm{s}$ and the airflow rate was $550 \mathrm{~g} / \mathrm{s}$, and the corresponding overall equivalence ratio was 0.46 . Simulations with and without turbulence forcing at the fuel/air inlets were performed, and its effect on numerical results was investigated. For turbulence forcing, a spectral synthesizer method from
Smirnov et al., [24] was used. In this method, fluctuating velocity components are computed by synthesizing a divergence-free velocity-vector field from the summation of Fourier harmonics based on the turbulence intensity of $7.5 \%$ at fuel and air inlets. The inlet temperature of fuel and air was $293 \mathrm{~K}$.

At wall boundaries, when the mesh was fine enough to resolve the laminar sub-layer, the wall shear stress was obtained from the laminar stress-strain relationship, and if the mesh was coarse the logarithmic law-of-the-wall was employed. That is, an enhanced wall boundary treatment was employed, where the turbulent and laminar wall laws were smoothly combined by a blending function [16]. As noted by Piomelli et al., [25], the boundary conditions correlating wall shear stress and velocity in the logarithmic layer were well suited to large eddy simulations in which the small scales were eliminated by filtering. At the combustor exit, an atmospheric pressure was specified.

For accurate calculation of the temperature field, the heat losses through the combustor walls and flame-holder body as shown in Fig. (1) had to be properly accounted for. In the present study, the mean temperature profiles obtained from the previous RANS simulation [12] were used to define the thermal conditions at these wall boundaries. It was known that the thermal inertia of the combustor wall and flameholder body was much larger than those of random turbulence eddies adjacent to the wall. This implied that the instantaneous heat flux through the wall could vary with time and location, depending on the temperature of the wall and turbulence eddies. The time averaged heat flux should be consistent with those in the RANS simulations. In this way, the nature of the heat transfer between the flow field and combustor walls could be adequately predicted.

For the RANS simulation in reference [12], not only the combustor flow field but also the solid regions of the combustor and insulation walls, and flame-holder body were meshed. That is, the conjugate heat transfers between the solid regions and flow field were computed. Moreover, the radiation heat transfer between the burned gas mixture and solid walls was included in the simulation. The velocity, temperature and major species fields in the combustor were reasonably well predicted with respect to the experimental database. The mean temperature profiles at all these wall boundaries were exported and read into the LES simulation as their thermal boundary conditions.

\subsection{Solution Methods}

A segregated solver with a bounded second-order accurate central difference scheme, a second-order implicit transient formation, and the SIMPLEC pressure-velocity coupling was used to resolve the flow field. The time step was first estimated using the following expression:

$$
\Delta t \leq(\Delta /|U|)_{\min }=\left(V^{1 / 3} /|U|\right)_{\min }
$$

where $|\mathrm{U}|$ is the local velocity magnitude. This means, the resolved smallest eddy would not advance more than one cell for each time step. A few time step sizes were tried and finally a time step of $2 \mu \mathrm{s}$ was used for all simulations, and 
the maximum CFL in the computational domain was 0.75 . Twenty iterations were performed for each time step.

The influence of time step size and convergence criteria on LES results of a periodic flow over a 2-D hill was investigated by Kornlass, et al., [26]. They found that the differences of mean velocities and fluctuations were negligible for time step sizes corresponding to $\mathrm{CFL}=1,2$ and 5 and the convergence criteria of normalized residuals from 0.1 to 0.0001 . For the present study, the normalized residual is $\sim 3 \times 10^{-3}$ for axial velocity, $\sim 2 \times 10^{-4}$ for radial and tangential velocities, and $2-6 \times 10^{-6}$ for energy and species.

An 8-node LINUX cluster, 64-bit, 2.6GHz, 16GB RAM, four-cores for each node, was used to carry out all simulations. Data were collected after 10 flow-through-times ( $\mathrm{T}_{\text {flow, }}$, the combustor length divided by the axial velocity averaged over the whole combustor), and the sampling interval was $20 \mu \mathrm{s}$. The corresponding sampling rate was 50 $\mathrm{KHz}$ or a cut-off frequency of $25 \mathrm{KHz}$.

\section{RESULTS AND DISCUSSION}

In the following sub-sections, the effects of inlet flow perturbation and data sampling period on LES simulation are discussed first, and then some of the numerical results and comparisons with the experimental database are presented and discussed.

\subsection{Effects of Inlet Flow Perturbation and Data Sampling Period}

Shown in Fig. (3) are the time-average axial velocity and temperature profiles along the combustor centerline with and without inlet flow perturbation. These results are averaged over $4 \mathrm{~T}_{\text {flow. }}$. It is clear that the mean axial velocity and temperature profiles for both cases are almost identical. That is, the effect of imposed turbulence perturbation on the LES results is negligible for the present configuration. A Similar observation is also reported by Strakey \& Eggenspieler [8], where a lean premixed swirl burner was studied with LES, and the inlet flow perturbation was generated by the same method with a turbulent kinetic energy of $5 \%$ of the mean flow.

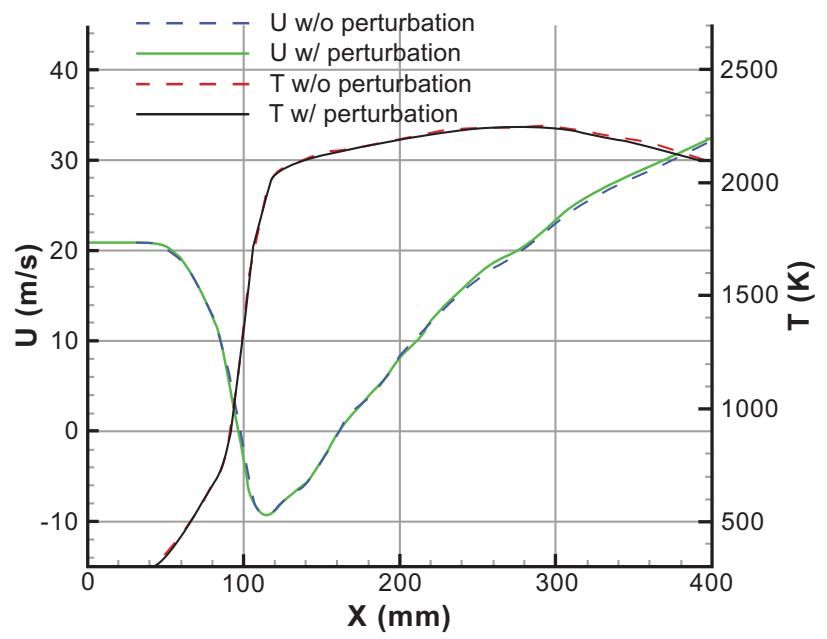

Fig. (3). Mean axial velocity and temperature profiles along the combustor centerline.

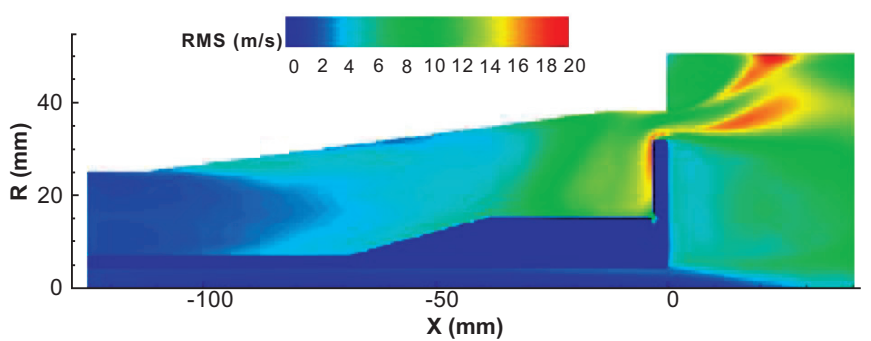

Fig. (4). RMS of velocity magnitudes in the upstream region.

It is anticipated that the above observation is attributed to the configuration of the present combustor. The contours of time averaged RMS (root mean square) of velocity magnitudes in the upstream of the combustor are shown in Fig. (4). It is clear that the imposed velocity fluctuations at the air inlet decay quickly and increase further downstream. The high turbulence level is observed around the flameholder due to the existence of a narrow annular slot between the flame-holder disc and the combustion wall. For the fuel supply line, the ratio of the length of the inlet section over the fuel tube diameter is 15.4 (Fig. 1), and therefore the imposed inlet turbulence is adjusted to a fully developed condition inside the tube before entering the combustor chamber. These may explain why the imposed inlet flow perturbation has negligible effect on the LES results in the present work.

Turbulence is characterized by randomness and involves a wide range of time and length scales [27]. In order to accurately capture the flow statistics, the data sampling frequency and sampling period have to be properly determined [28]. Since the grid size in LES is much smaller in comparison with the computational domain geometry, the computing time step is much short, for example, $2 \mu$ s for the present case. Therefore, there is no problem in satisfying the cut-off frequency requirement in the flow.

However, when the lowest frequency of interest in the flow is only a few $\mathrm{Hz}$, in order to resolve this frequency (collect enough independent numerical data points) with an acceptable accuracy, the total sampling time or computing flow time should be at least dozens of times longer than the period of this frequency. As a result, the required computing power and time can be extremely costly. For the present case, the experimental measurements and observations suggest that there are large-scalar (such as high temperature) eddies randomly moving in the circumferential direction with size comparable to the combustor radius and a frequency close to a few $\mathrm{Hz}$.

Fig. (5) shows the mean axial velocity and temperature profiles along two radial directions at the intersection of $\mathrm{X}=$ $80 \mathrm{~mm}$ and $\mathrm{Y}=0 \mathrm{~mm}$, where the $\mathrm{Y}$ axis is perpendicular to the $\mathrm{X}$ axis. The results are averaged over $9 \mathrm{~T}_{\text {flow }}$ at about 19 $\mathrm{T}_{\text {flow }}, 0.467 \mathrm{~s}$. The mean negative axial velocity observed in Fig. (4) indicates that this section is located in the recirculation zone behind the flame-holder. Fig. (5) shows that although the flow-through-time is about 0.5 second and the geometry, mesh and fuel/air supplies are exactly axisymmetric, deviations in mean flow parameters along the circumferential direction still exist. This suggests that the large-scale eddies with large time scales are not completely 
resolved. Fortunately, the differences are limited and for engineering purposes they are considered acceptable.

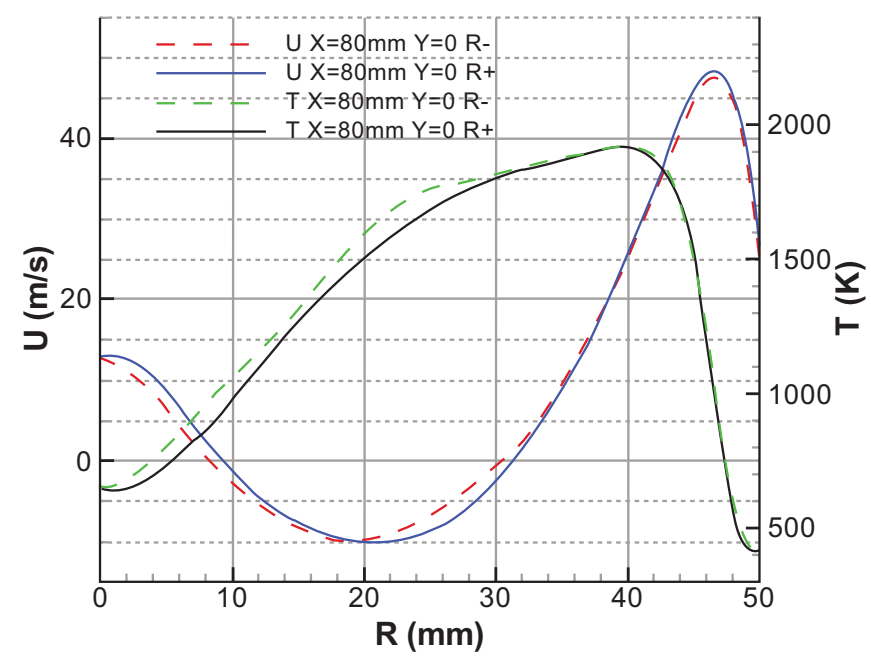

Fig. (5). Mean axial velocity and temperature profiles at $X=80$ $\mathrm{mm}$ and $\mathrm{Y}=0 \mathrm{~mm}$.

Considering the above sampling statistics requirement, a second data set with a total flow time of 7 was obtained. To increase effective sampling time, the radial distributions of mean flow parameters are averaged over 60 planes along the circumferential direction of the combustor. It is expected that the statistics of flow parameters are significantly improved. These averaged flow results are presented in the following sub-sections.

\subsection{Velocity Distributions}

The top portion of Fig. (6) is a snapshot of instantaneous axial velocity contours along the longitudinal plane $\mathrm{Y}=0$ $\mathrm{mm}$, and the middle and bottom of the figure are snapshots of axial velocity contours at 8 axial cross-sections from $\mathrm{X}=$ $20 \mathrm{~mm}$ to $200 \mathrm{~mm}$. The chaotic nature of turbulence is clearly illustrated by both plots. The high velocity air enters the combustor chamber through the gap between the flameholder and the inlet section wall (see Fig. 1), and moves outwards and then along the chamber wall. Less variation of axial velocities is observed just behind the flame-holder and far downstream $X>200 \mathrm{~mm}$. The regions with negative axial velocities are the recirculation zones, and they almost disappear downstream of $X \approx 150 \mathrm{~mm}$, where the stagnation or reattachment point is expected. The size of eddies is gradually increased behind the flame-holder, large eddies are observed in the range of $X=\sim 50 \mathrm{~mm}$ to $\sim 100 \mathrm{~mm}$, then start to break-up, and eventually are broken into small pieces far downstream.

The unsteady nature of LES provides information to understand complex physical phenomena in the combustor chamber, such as coherent structure, vortex shedding, correlations, power spectrums, etc. These phenomena can not be revealed by RANS simulations. A critical phenomenon to combustion stability, dynamics of recirculation zones, is briefly described here, and detailed analyses of the unsteady phenomena in the combustor will be presented in another paper.

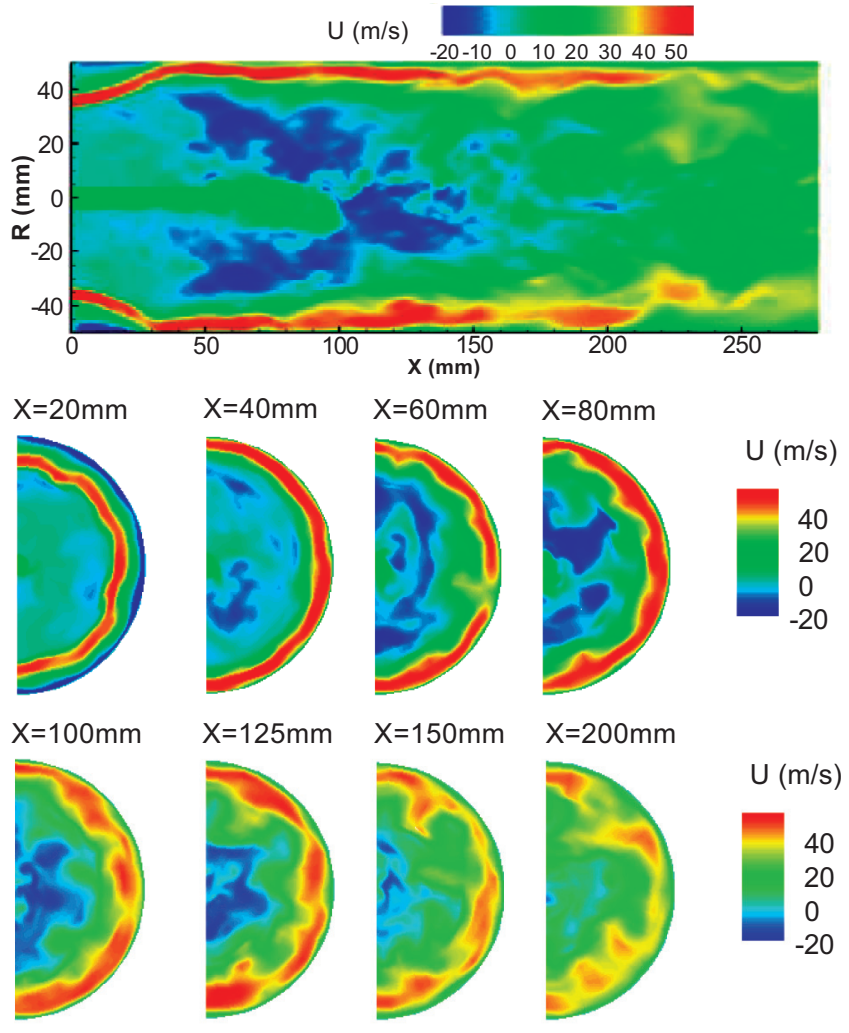

Fig. (6). Snapshots of axial velocity contours at $\mathrm{Y}=0 \mathrm{~mm}$ and eight cross-sections $X=20-200 \mathrm{~mm}$.
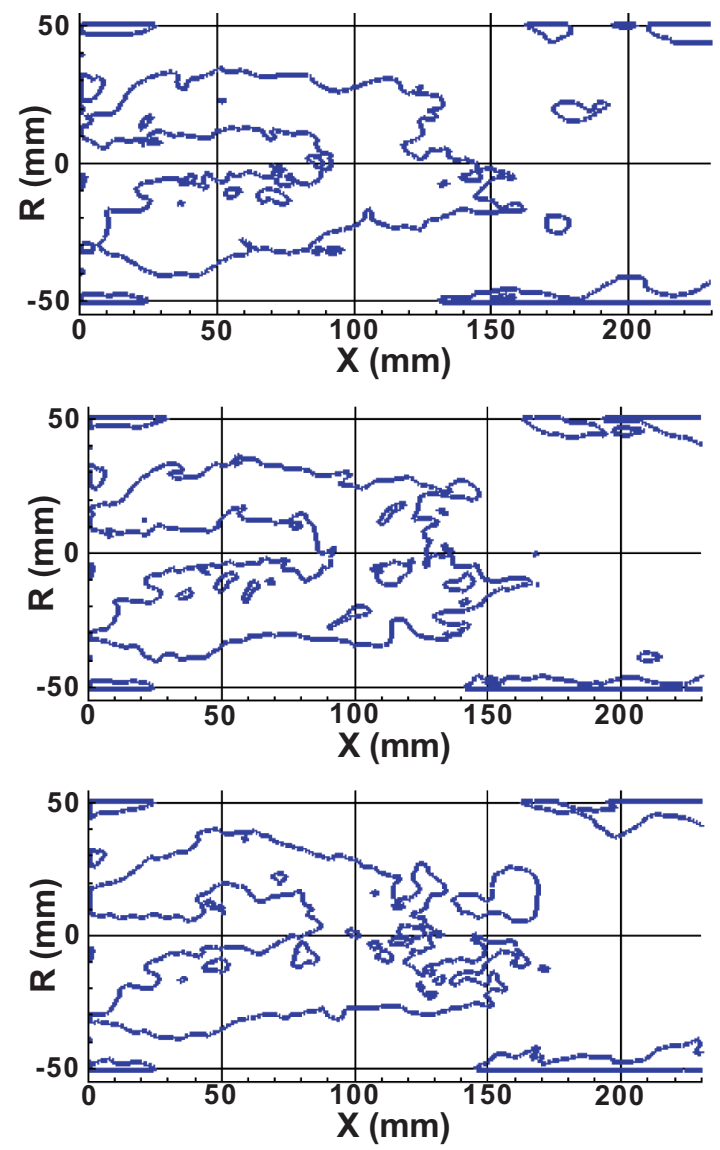

Fig. (7). Vortex shedding behind the flame-holder. 
Zero contour lines of instantaneous axial velocities are shown in Fig. (7) at $\mathrm{Y}=0 \mathrm{~mm}$ plane for three time instants. Two recirculation zones behind the flame-holder are expected: one created by the fuel jet and the other by the annular air jet, as shown by all three plots. The recirculation zone formed by the fuel jet is so weak, and as a result, it could not penetrate the large recirculation zone formed by the air jet. This is consistent with the experimental measurements and the RANS results [12].

The size of the recirculation zones varies with time. The middle plot is $0.6 \mathrm{~ms}$ later than the top one; while the third is $0.88 \mathrm{~ms}$ after the middle plot. More small eddies or recirculation zones are observed in the middle plot than in the top one. The third clearly shows that a relatively large piece of zero-axial velocity pocket is torn away from the large recirculation zone, the downstream region is broken into many branches, and the volume of the recirculation zone is reduced. Variation of the shape and volume of the center recirculation is also observed, although it is not as significant as in the case of the large recirculation zone.

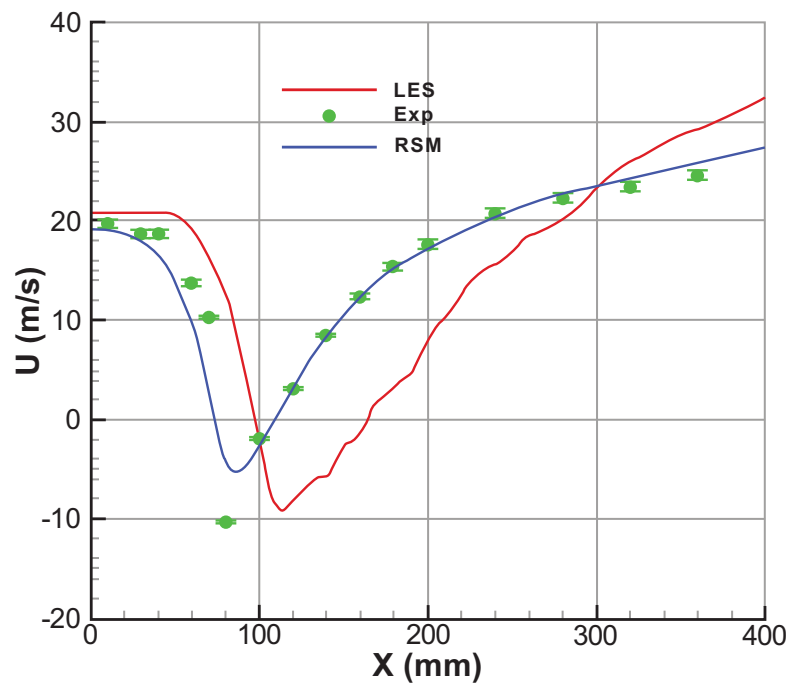

Fig. (8). Mean axial velocity along the combustor centerline.

Fig. (8) gives the mean axial velocity profiles along the combustor centerline for the LES and the optimized RANS simulation [12]. Superimposed in the figure are the experimental measurements with an estimated error of $2 \%$. For the RANS results, the trends and magnitudes of numerical results are consistent with the experimental data. For the LES, large deviations are observed in the middle region for the LES. The magnitude of the maximum negative velocity is adequately predicted by the LES, but underpredicted by the RANS.

The predicted mean axial velocity profiles at five crosssections, two inside the recirculation region, one close to the stagnation point, and the last two located downstream of the recirculation region, are presented in Fig. (9) for both LES and optimized RANS, and quantitatively compared with the experimental database. In general, the profiles are reasonably predicted, except in the local regions at $\mathrm{X}=40 \mathrm{~mm}$ and $\mathrm{X}=$ $160 \mathrm{~mm}$ for LES, and $X=40 \mathrm{~mm}$ and $100 \mathrm{~mm}$ for RANS. It should be mentioned that the flow field is complex at these sections, and consequently the numerical simulation is particularly challenging. The LES shows better performance at three upstream sections, while the RANS is better at two downstream sections.

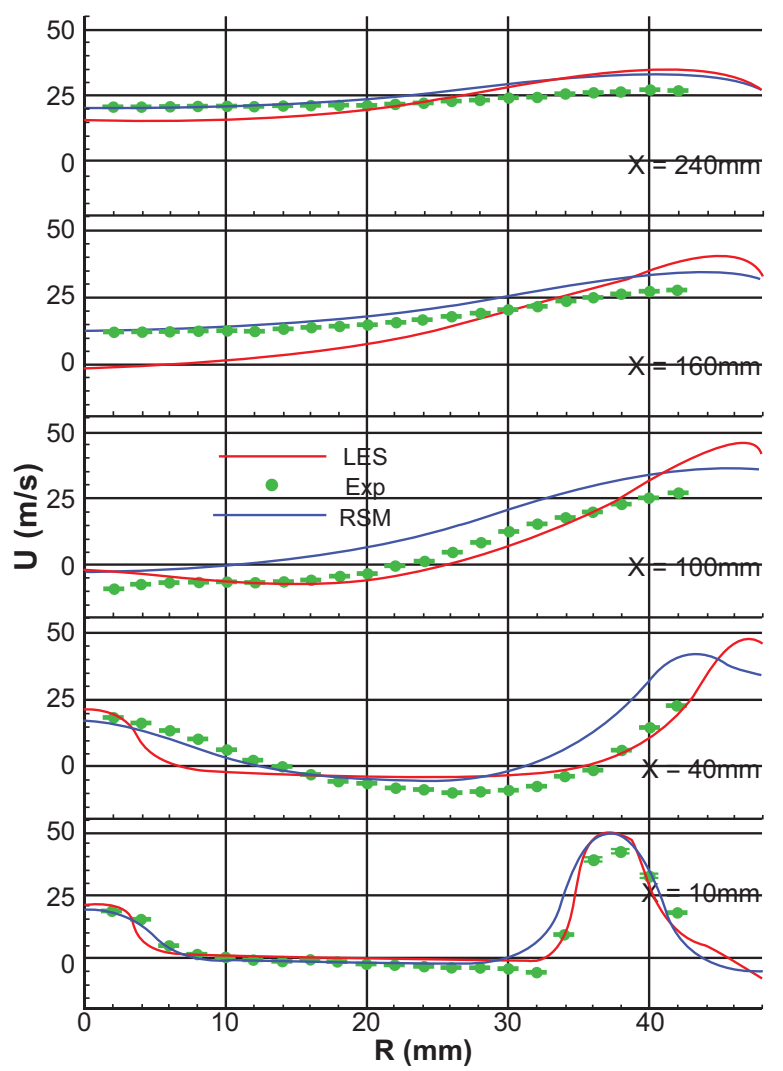

Fig. (9). Mean axial velocity profiles at sections $X=10-240 \mathrm{~mm}$.

\subsection{Temperature Distributions}

In Fig. (10), a snapshot of temperature contours at $\mathrm{Y}=0$ $\mathrm{mm}$ is shown on the top portion, and on the middle and bottom the instantaneous temperature contours at 8 axial cross-sections are displayed. Similar to Fig. (6), the chaotic nature of turbulence is illustrated. These contour plots indicate that intense chemical reaction first takes place near the edge of the flame-holder around the envelope of the recirculation zone, where the fresh air mixes with the hot gas mixture and burns. The chemical reaction or hightemperature region propagates downstream outwards and inwards and eventually reaches the combustor centerline at $\mathrm{X} \approx 125 \mathrm{~mm}$. The temperature near the combustor center gradually increases from $X=20 \mathrm{~mm}$ to $200 \mathrm{~mm}$.

The predicted temperature profiles along the combustor centerline for both LES and RANS are compared with the experimental data in Fig. (11), where the measurement error is about $5 \%$. In general, the trends and magnitudes of the numerical results are close to the measurements. The rising portion of the mean temperature profile is consistent with the experimental data. That is, the chemical reaction propagation from the flame-holder edge towards the combustor centerline is fairly well predicted. However, the mean temperature is over-predicted for $\mathrm{X}>120 \mathrm{~mm}$ downstream for LES and in the middle portion $(X \approx 120-300 \mathrm{~mm}$ ) for RANS. Fig. (12) presents the temperature profiles at five cross-sections from $\mathrm{X}=52$ to $233 \mathrm{~mm}$. Again, the trends and magnitudes are 
consistent with the experimental observations, except in the center region at $\mathrm{X}=82 \mathrm{~mm}$ for LES. This is because the propagation of chemical reaction towards the combustor center is little slower than the experimental observation near $\mathrm{X}=82 \mathrm{~mm}$.

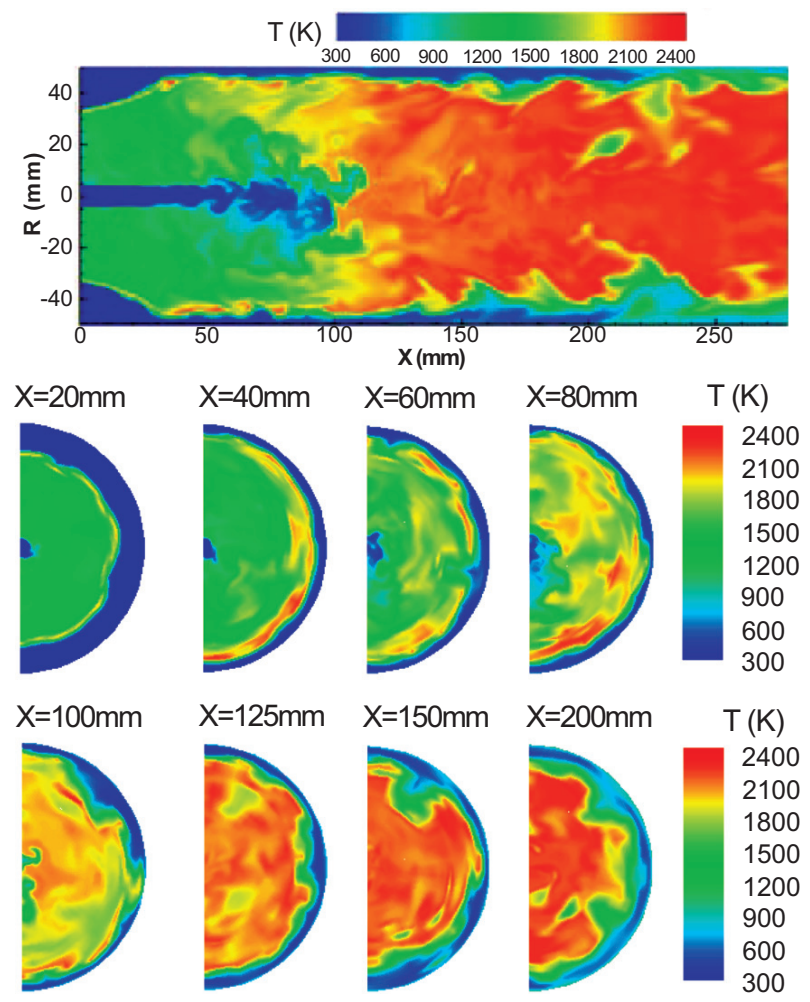

Fig. (10). Snapshots of temperature contours at $\mathrm{Y}=0 \mathrm{~mm}$ and eight axial cross-sections $X=20-200 \mathrm{~mm}$.

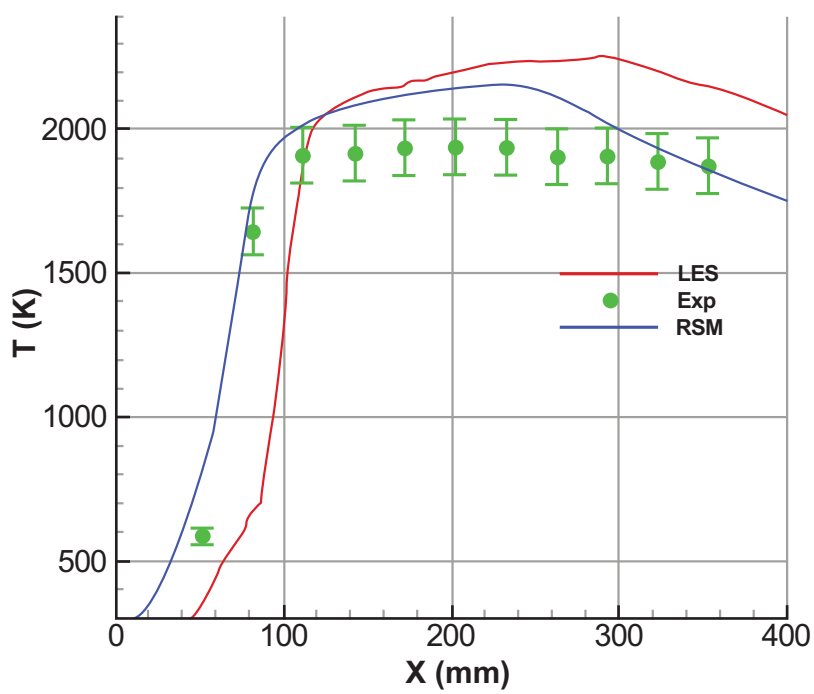

Fig. (11). Mean temperature profile along the combustor centerline.

In Figs. (11, 12), it is found that the predicted temperature in the high temperature region is higher than the measured values. The maximum difference is about $200 \mathrm{~K}$. One of the anticipated reasons is that the temperature was measured by a $0.25-\mathrm{mm}$ diameter thermocouple. Owing to the radiation and conduction losses from the thermocouple, the measurement error could exceed $100 \mathrm{~K}$ over regions where the gas temperature was high and the flow velocity was low [29].

In addition, the one-step chemical reaction employed in the present work can contribute to this observed discrepancy although the effect is expected relatively minor in comparison with the first one according to a benchmark study on four combustion models on this combustor [20].

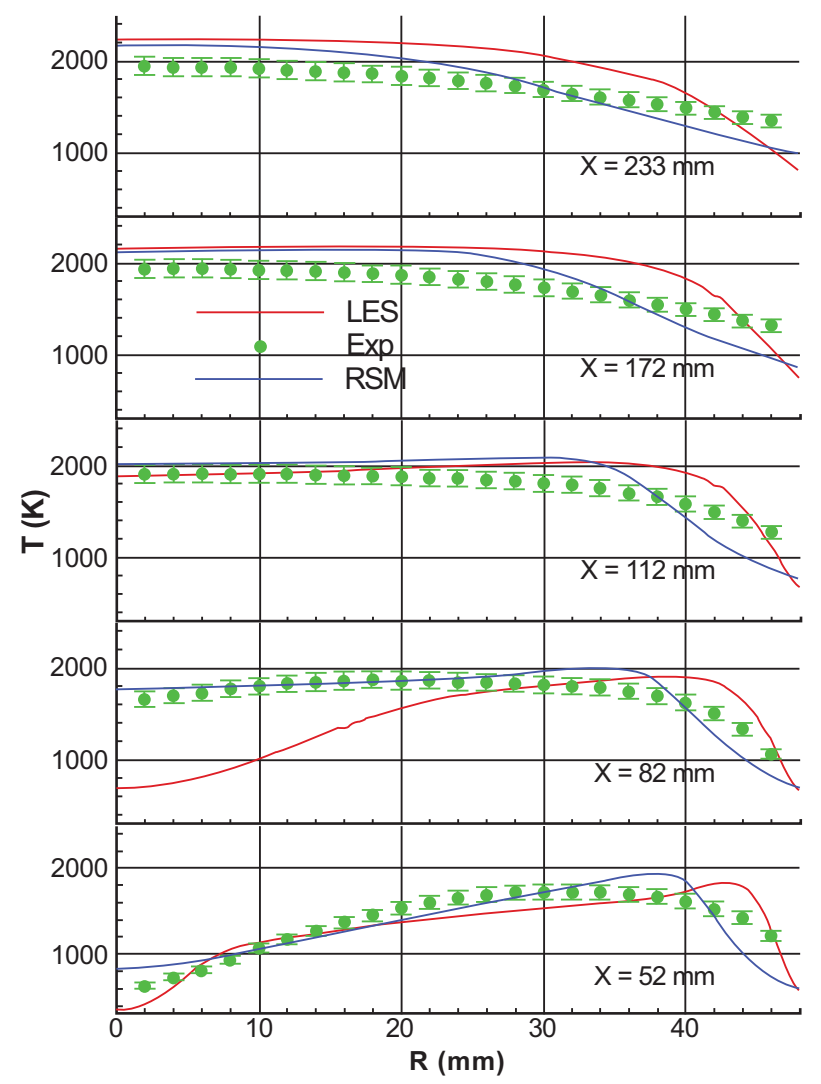

Fig. (12). Mean temperature profiles at sections $X=52-233 \mathrm{~mm}$.

\section{DISCUSSION}

As shown in the above results, the mean axial velocity and temperature can be reasonably predicted by LES, except for some local regions. In terms of validation, the quality of LES prediction in the present work is comparable to those from James' swirl-stabilized model combustor [2] and Mahesh's co-axial combustor [3]. All these indicate that the currently accepted engineering LES approaches are promising for simulating combustion systems. However, the comparisons of the LES results with the experimental database suggest that further improvement of these approaches is required in order for LES to play a reliable role in the development of advanced combustion systems.

Ten fundamental questions concerning large eddy simulations in turbulent flows have been raised by Pope [30]. Adequate discussion of these questions is outside the scope of the present work. Two of Pope's ten points are closely related to grid size in the computational domain. Fig. (13) shows contours of a grid factor II, $\mathrm{R}_{2}$ that is defined as the ratio of the local grid size to the local Kolmogorov turbulence length scale [31] 
$R_{2}=\Delta / \eta=V^{1 / 3} /\left(v^{3} / \varepsilon\right)^{1 / 4}$

where $\eta$ is the Kolmogorov length scale and $v$ is the molecular kinematic viscosity. In Fig. (13), Kolmogorov length scales are estimated from the same RANS simulation as that for Fig. (2), and the temperature dependent molecular kinematic viscosity is used. Fig. (13) shows that the grid size is about 5-7 times larger than the local Kolmogorov scale near the flame-holder and in the vicinity of the combustor wall and about 2-5 times near the combustor centerline downstream of $X \approx 50 \mathrm{~mm}$. That is, the grid resolution is good enough for flow field LES; however its effect on combustion or molecular mixing and chemical reaction should be further investigated since they occur at the smallest scales.

Another important reason for the above observed discrepancies is that much effort has been devoted to the development of RANS turbulence models in the last five decades, and these models and their coefficients have been extensively validated or calibrated against experimental results. In contrast, the LES is relatively new and costly in computing power and time. As a result, detailed studies and validations are limited.

As mentioned by Pitsch [1] and James et al., [2], although the important issues related to LES have been visited by many researchers, some fundamental questions of combustion LES remain to be addressed, and more validation and verification efforts are required in order to make the LES a valuable and cost-effective tool for the design of combustion devices. The present study supports their opinions.

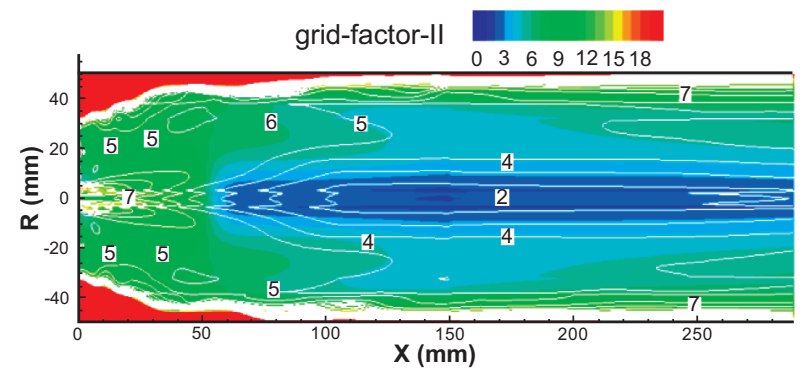

Fig. (13). Grid factor $\mathrm{R}_{2}$ estimated from RANS simulation.

\section{CONCLUSIONS}

An attempt at large eddy simulations in a benchmarking diffusion-flame model combustor has been made. Because of the mixing control nature of diffusion flames, the uncertainty of combustion modeling on LES results is expected to be minimized. The mesh size, inlet perturbation, wall boundary conditions, physical sub-models, time step and total sampling time have been selected or discussed.

The LES results are encouraging and the comparisons with the benchmarking database indicate that LES can provide reasonable predictions for the mean axial velocity and temperature distributions inside the combustor chamber. The air/fuel inlet turbulence forcing does not affect the LES results for the present configuration, and the turbulence can develop naturally in the combustor inlet section. The present study also suggests that in order to capture the lowest frequency of interest in the flow, the computing flow time should be long enough.

In order to make LES a valuable and cost-effective tool in the development of advanced combustion systems, many fundamental questions should be revisited and more validation efforts are required.

\section{ACKNOWLEDGEMENTS}

The authors are grateful to Dr. Bill Wallace for his valuable comments and suggestions during proofreading.

\section{CONFLICT OF INTEREST}

Declared none.

\section{NOMENCLATURE}

$\mathrm{D}=$ Molecular diffusivity

$\mathrm{k} \quad=$ Turbulence kinetic energy

$1=$ Energetic turbulence length scale

$\mathrm{p} \quad=$ Pressure

$\mathrm{R} \quad=$ Radial direction

$\mathrm{R}_{1}=$ Grid factor I

$\mathrm{R}_{2}=$ Grid factor II

$\mathrm{S}_{\mathrm{ij}}=$ Strain rate tensor

$\mathrm{T}=$ Temperature

$\mathrm{T}_{\text {flow }}=$ Flow-through-time, the combustor length divided by the axial velocity averaged over the whole combustor

$\mathrm{U} \quad=$ Mean axial velocity

$\mathrm{V}=$ Cell volume

$\mathrm{X}=$ Axis along the combustor axis of symmetry

$\mathrm{Y}=$ Axis perpendicular to $\mathrm{X}$ axis

\section{Greek Letters}

$\varepsilon=$ Turbulence dissipation rate

$\rho \quad=$ Density

$\Delta \quad=$ Local grid size

$\mu \quad=$ Molecular viscosity

$\eta \quad=$ Kolmogorov turbulence length scale

$v \quad=$ Molecular kinematic viscosity

$\phi \quad=$ Species or total enthalpy

\section{REFERENCES}

[1] H. Pitsch, "Large-Eddy Simulation of Turbulent Combustion", Annu. Rev. Fluid Mech., Vol. 38, pp. 453-482, 2006.

[2] S., James, J. Zhu, and M.S. Anand, "Large-Eddy Simulation as a Design Tool for Gas Turbine Combustion Systems", AIAA J., Vol. 44, No. 4, pp. 674-686, 2006.

[3] K. Mahesh, G.. Constantinescu, S. Apte, G., Iaccarino, F. Ham, and P. Moin, "Large-Eddy Simulation of Reacting Turbulent Flows in Complex Geometries", J. Appl. Mech., Vol. 73, pp. 374-381, 2006. 
[4] S.M. Cannon, B. Zuo, and C.E. Smith, "LES Prediction of Combustor Emissions from a Practical Industrial Fuel Injector", ASME GT2003-38200, 2003.

[5] G.. Boudier, L.Y.M. Gicquel, and T.J. Poinsot, "Effects of Mesh Resolution on Large Eddy Simulation of Reacting Flows in Complex Geometry Combustors", Combust. Flame, Vol. 155, pp. 196-214, 2008.

[6] B. Gherman, R.Z. Szalo, and L. Fuchs, "LES of Swirling Flows in Gas Turbine Combustion Chamber", ASME GT2004-53711, 2004.

[7] W.W. Kim, J.J. Lienau, P.R.V. Slooten, III, M.B. Colket, R.E. Malecki, and S. Syed, "Towards Modeling Lean Blow Out in Gas Turbine Flame-Holder”, ASME GT2004-53967, 2004.

[8] P. Strakey, and G. Eggenspieler, "Development and Validation of a Thickened Flame Modeling Approach for Large Eddy Simulation of Premixed Combustion", ASME GT2009-60077, 2009.

[9] G.. Kunne, C. Klewer, and J. Janicka, "Hybrid RANS/LES Simulation of a Lean Premixed Swirl Burner Based on a Turbulent Flame Speed Closure", ASME GT2009-59443, 2009.

[10] I.Campbell, and D.L. Logan, "An Experimental Study of a Combusting Flow Past a Confined Bluff Body", Combustion Institute - Canadian Section, Halifax, Canada, 1997.

[11] R.W. Bilger, "Turbulent Diffusion Flames", Annul. Rev. Fluid Mech., Vol. 21/101, pp. 101-135, 1989.

[12] L.Y. Jiang, and I. Campbell, "Reynolds analogy in Combustor Modeling", Int. J. Heat Mass Transf., Vol. 51/5-6, pp. 1251-1263, 2008.

[13] D.C. Wilcox, Turbulence Modeling for $C F D, 2^{\text {nd }}$ ed., DCW Industries, Inc., La Canada California, p. 118, 2002.

[14] S.E. Gant, "Reliability Issues of LES-Related Approaches in an Industrial Context", Flow Turbulence Combust, Vol. 84, pp.325335,2010 .

[15] G.. Boudier, L.Y.M. Gicquel, and T.J. Poinsot, "Effects of Mesh Resolution on Large Eddy Simulation of Reacting Flows in Complex Geometry Combustors", Combust. Flame, Vol. 155, pp. 196-214, 2008.

[16] ANSYS Inc., "ANSYS Fluent 12.0 Documentation”, 10 Cavendish Court, Lebanon, NH 03766, USA, 2009.

[17] W.W. Kim, and S. Menon, "Application of the Localized Dynamic Sub-Grid-Scale Model to Turbulent Wall-Bounded Flows", AIAA paper, 97-0210, 1997.

[18] P. Moin, W. Squires, W. Cabot, and S. Lee, "A Dynamic Sub-GridScale Model for Compressible Turbulence and Scalar Transport", Phys. Fluids A 3 (11), pp. 2746-2757, 1991.
[19] H. Pitsch, and H. Steiner, "Large-Eddy Simulation of a Turbulent Piloted Methane/Air Diffusion Flame", Phys. Fluids, Vol. 12, No. 10, pp. 2541-2554, 2000.

[20] L.Y. Jiang, and I. Campbell, "Combustion Modeling in a Model Combustor", J. Aerospace Power, Vol. 22, No. 5, pp. 694-703, ISSN 1000-8055, 2007.

[21] B.F. Magnussen, and B.H. Hjertager, "On mathematical Models of Turbulent Combustion with Special Emphasis on Soot Formation and Combustion", In: 16th Symposium on Combustion (International), pp. 719-729, 1976.

[22] L.Y. Jiang, and I. Campbell, "Radiation Benchmarking in a Model Combustor", J. Eng. Gas Turbine Power, Vol. 131, Jan. 2009, 011501.

[23] J.W. Rose, and J.R. Cooper, Technical Data on Fuel, John Wiley \& Sons, New York, 1997.

[24] R.. Smirnov, S. Shi, and I. Celik, "Random Flow Generation Technique for Large Eddy Simulations and Particle-Dynamics Modeling", Trans. ASME, J. Fluids Eng., Vol. 123, pp. 359-371, 2001.

[25] U. Piomelli, J. Ferziger, and P. Moin, "New Approximate Boundary Conditions for Large Eddy Simulations of WallBounded Flows", Phys. Fluid A 1(6), pp. 1061-1068, 1989.

[26] M. Kornlass, D.C. Sternel, and M. Schafer, "Influence of Time Step Size and Convergence Criteria on Large Eddy Simulations with Implicit Time Discretization", in Quality and Reliability of Large-Eddy Simulations, J. Meyers, et al. (Eds.), Springer, Netherlands, 2008.

[27] H. Tennekes, and J.L. Lumley, A First Course in Turbulence, the MIT Press, Cambridge, Mass, 1983.

[28] J.S. Bendat, and A.G.. Piersol, Random Data: Analysis and Measurement Procedures, $2^{\text {nd }}$ ed., John Wiley \& Sons, New York, 1986.

[29] J.P. Sislian, L.Y. Jiang, and R.A. Cusworth, "Laser Doppler Velocimetry Investigation of the Turbulence Structure of Axisymmetric Diffusion Flames," Prog. Energy Combust. Sci. 14, pp. 99-146, 1988.

[30] S.B. Pope, "Ten Questions Concerning the Large-Eddy Simulation of Turbulent Flows," New J. Phys. 6, Vol. 35, 2004.

[31] J.O. Hinze, Turbulence, the McGraw-Hill Book Company Inc., New York, p.223. 1987. 